\title{
INFLUENCIA DE LAS REVISTAS DE IMPACTO EN EL PERIODISMO CIENTÍFICO Y EN LA CIENCIA ACTUAL
}

\author{
Carlos Elías
}

Universidad Carlos III de Madrid

\begin{abstract}
RESUMEN
En este estudio se ha intentado detectar la influencia de las revistas de impacto, en las que mayoritariamente publican los científicos, en el periodismo especializado y en la ciencia actual. He analizado sobre todo el caso español, pero considero que sus conclusiones pueden extenderse al resto de los países. Al margen de que se demuestra la absoluta preponderancia de revistas como Nature y Science: el $45 \%$ de la información sobre ciencia de científicos extranjeros que publican los diarios generalistas procede de estas revistas, también se analizan las influencias de la estrategia de comunicación mediática de los gabinetes de prensa de estas publicaciones en la ciencia. Se ha estudiado especialmente la preponderancia del idioma inglés y la escasa relevancia del español, así como las consecuencias futuras que tendrá el cada vez mayor impacto de unas pocas revistas en la modificación del método científico en las ciencias experimentales.
\end{abstract}

\section{ANÁLISIS CUANTITATIVO}

Se ha estudiado un periodo de seis meses: febrero, marzo, abril, mayo, junio y julio de 1998. Se han seleccionado los diarios de tirada nacional: $E l$ Pais, El Mundo, Abc, Diario 16, El Periódico de Cataluña y La Vanguardia. En total se han encontrado 1.458 noticias sobre ciencia. En muchas, la fuente era española y simplemente se la consultaba respecto a una información de la que ese científico no era responsable, sino experto en esa área. De esas noticias, 226 provenían de trabajos publicados en revistas científicas de impacto. De 
forma que el $15,5 \%$ de toda la información sobre ciencia que se publica en España proviene de estas revistas. También se ha obtenido otro dato: el 45\% de las noticias cuya fuente es un científico o centro extranjero proviene de información elaborada a partir de revistas de difusión científica.

Debe mencionarse otro resultado relevante: en las 1.458 informaciones que he revisado no aparece ninguna que se base en los resultados publicados en una revista científica española o en idioma español ${ }^{1}$.

Debo aclarar que el único estudio parecido que he encontrado en la bibliografía es uno elaborado por el Observatorio de Comunicación Científica de Barcelona ${ }^{2}$. En él se incluyeron 1.060 noticias procedentes de seis diarios europeos y uno estadounidense y, tras su análisis, se concluyó que el $25 \%$ de las mismas procede de revistas científicas. Aún resulta más sorprendente que el $62 \%$ de esas informaciones que provenían de revistas lo hacía de cuatro cabeceras: Nature, Science, The Lancet y British Medical Journal.

Este trabajo, centrado exclusivamente en prensa española, nos da un porcentaje sensiblemente inferior al del estudio catalán en 9,5 puntos porcentuales, lo que supone un 38\% menos del resultado del trabajo del Observatorio de Comunicación Científica de la Universidad Pompeu Fabra de Barcelona. Esta diferencia en el resultado podría explicarse, aunque no en este caso concreto, porque de forma coyuntural la información publicada en nuestro periodo de estudio tuvo un mayor carácter nacional. Éste es uno de los problemas de los estudios cuantitativos de los medios de comunicación: que todos los periodos históricos no son iguales.

En este caso se están comparando dos resultados que han sido obtenidos siguiendo métodos de selección de las noticias diferentes. En este sentido, una de las diferencias del estudio presentado en este artículo es el hecho de que en este $15,5 \%$ no se incluyen las 94 noticias que también citan una revista extranjera pero que, como la fuente es un científico del Consejo Superior de Investigaciones Científicas, las he eliminado ${ }^{3}$ del cómputo general. Si las incluyéramos, obtendríamos 320 informaciones procedentes de revistas, de las 1.458 totales. Es decir, que podríamos afirmar que el 21,9\% de la información científica que se publica en España procede de estas revistas de referencia.

${ }^{1}$ Lo único que suele aparecer, sobre todo en el suplemento Salud de El Mundo, son informaciones referidas a resultados que se han leído en una tesis doctoral - todas se refieren a tesis doctorales leídas en la Facultad de Farmacia de la Universidad Complutense-; sin embargo, no podemos presuponer que estos resultados los publicarán en una revista española, sino más bien al contrario.

${ }^{2}$ Este estudio ha sido citado por Cristina Ribas en el artículo «La influencia de los comunicados de prensa, según el color del cristal con que se mire», revista Quark, Ciencia, Medicina, Comunicación y Cultura, n. ${ }^{\circ}$ 10, enero-marzo 1998 (pp. 32-37).

${ }^{3}$ Se ha eliminado porque el interés de la investigación residía en estudiar el impacto de las revistas especializadas en la prensa generalista. Las noticias publicadas correspondientes al CSIC se insertan a través del gabinete de prensa de este organismo, que tiene aún mayor influencia mediática que las revistas especializadas. 
Aun así, este valor es inferior al del Observatorio de Comunicación Científica. Esto podría explicarse por el hecho de que nosotros hemos incluido informaciones de opinión en el cómputo general y, sobre todo, porque los periódicos europeos y estadounidenses hacen más uso de los comunicados de prensa que los españoles, básicamente porque éstos están escritos en inglés y, en especial, porque los científicos que publican en las revistas de referencia son, sobre todo, de los países de procedencia de los diarios estudiados por los investigadores catalanes. No hay que olvidar que la cercanía de la fuente es un buen argumento para publicar una información científica.

Prefiero quedarme con la cifra del $15,5 \%$ porque es más puro. Es decir, ese porcentaje corresponde a informaciones que literalmente copian el comunicado de prensa, por lo que no sólo han sido inducidas por ellos sino que, básicamente, son una copia de los mismos, de forma que los criterios de selección de la información no dependen de los periodistas científicos, sino de los redactores de los gabinetes de prensa de las revistas de referencia.

De ese $15,5 \%$ total que procede de revistas científicas, el $50 \%$, es decir, la mitad, cita a Nature $(37,6 \%)$ o a Science (12,4\%), editada por la Asociación Americana para el Avance de la Ciencia. La siguiente revista en ser citada es The Lancet, que aparece en un $8 \%$ del total de noticias que proceden de revistas científicas. Le sigue a gran distancia Proceedings (4,8\%), una publicación editada por la Academia Nacional de Ciencias de Estados Unidos.

El resto de las revistas especializadas no aparecen en cantidad significativa y no las he incluido en los porcentajes porque, en general, aparecen asociadas a medios concretos. Así, por ejemplo, el suplemento de Salud del diario El Mundo suele incluir bastante información procedente de la New England Journal of Medicine, una publicación que apenas es citada en otros medios. Entre las revistas que, al menos, aparecen más de dos veces en nuestro estudio están, además de las ya mencionadas, Journal of American Medical Association (JAMA), Journal of The Royal Astronomy Society, Journal of the National Cancer Institute, Neuron, Applied Physics Letters, Journal of Virology, British Medical Journal, Journal of Human Genetics, Circulation, Cell y Journal of Biological Chemistry. Debe matizarse que las publicaciones satélites ${ }^{4}$ de Nature se han contabilizado como pertenecientes a la revista matriz. Sin embargo, debo señalar que las satélites de Nature apenas aparecen en los medios de comunicación.

${ }^{4}$ En el momento de redactar este artículo las publicaciones satélites de Nature eran: Nature Genetics, Nature Medicine, Nature Biotechnology, Nature Neuroscience, Nature Cell Biology y Nature Structural Biology. 


\section{INTERPRETACIONES CUALITATIVAS DEL ANÁLISIS CUANTITATIVO}

Tras estos resultados caben, en principio, dos reflexiones. La primera, a la que ya he hecho referencia y que mencionaré más adelante, es que no aparece ninguna publicación científica española o en el idioma español. La segunda, que la mayoría de las revistas pertenecen al ámbito de la medicina. Resalta el escaso eco que en la prensa tienen las revistas científicas especializadas en temas agrícolas y medioambientales, sobre todo porque este tipo de información cada vez se publica con más asiduidad, aunque con numerosas deficiencias que deberían ser estudiadas más a fondo5. Sí aparece, no obstante, algún informe de la organización ecologista Greenpeace cuyos resultados difunde esta organización en su revista trimestral. Pero, obviamente, ésta no es una publicación científica, sino la voz institucional de la organización ecologista.

Otra reflexión que aparece tras los resultados de este estudio es que en el periodismo científico español sucede lo mismo que en otros países: las revistas más citadas y de mayor prestigio para los científicos no son las que más aparecen en prensa. Los primeros lugares del Science Citation Index (SCI) los ocupan revistas de revisión, los denominados Abstracts, que aunque no publiquen noticias importantes resultan fundamentales para escribir reportajes sobre en qué situación se encuentra un tema. Tras los Abstracts, la publicación especializada mejor clasificada en el SCI es Cell, cuyos resultados apenas aparecen en 3 de 1.458 informaciones estudiadas.

\section{LOS GABINETES DE PRENSA EN LAS REVISTAS ESPECIALIZADAS}

A pesar de la calidad y la importancia de los trabajos que ofrecen muchas revistas especializadas, los periodistas españoles, al igual que los europeos, no las utilizan y prefieren revistas como Nature o Science, que, aunque con índices bastante altos ${ }^{6}$, son relativamente bajos en comparación con las más influyentes dentro de la misma ciencia.

La explicación está en que estas dos revistas poseen gabinetes de prensa muy profesionales que redactan en lenguaje periodístico los contenidos princi-

5 Un estudio de Miguel Montaño, de la Universidad de Sevilla, publicado en el n. ${ }^{\circ} 2$ (enero-junio 1999) de Ámbitos: Revista Andaluza de Comunicación, pp. 207-228, pone de manifiesto que las fuentes utilizadas en los diez primeros capítulos de un programa televisivo sobre Medio Ambiente, emitido por Canal Sur, proceden mayoritariamente de las empresas (25\%), la Administración (21\%) y las ONGs (19\%). Los científicos aparecen en cuarto lugar (17\%) y las revistas científicas internacionales especializadas en esos temas no aparecen como fuentes. Esta grave disfunción en la comunicación medioambiental debería ser estudiada de forma más profunda.

${ }^{6}$ No obstante, cada año los índices de impacto de estas revistas son más altos en virtud de un fenómeno que hemos denominado "Ciencia producida para salir en los periódicos», que supondrá en el futuro una perversión en el método científico y que expondré más adelante. 
pales de lo que publican ${ }^{7}$. Los periodistas no tienen que acceder así al artículo principal, redactado en un lenguaje de no fácil comprensión por la mayoría de los periodistas científicos españoles, lo cual facilita su trabajo. Además, al estar consideradas - no se sabe muy bien por qué, como explicaré más adelanteScience y Nature como fuentes institucionales u oficiales, la publicación de sus resultados no exige a los periodistas el contraste de la información, pues llevan el sello de la credibilidad, lo cual en términos empresariales facilita la producción periodística, puesto que se elimina el tiempo perdido en contrastar las fuentes. La sociedad es la que sale perdiendo, tal y como expresaba el periodista científico de The New York Times, Lawrence Altman, en una entrevista a la revista Quark, Ciencia, Medicina, Comunicación y Cultura $^{8}$, pues, en su opinión, el uso de información proveniente de los gabinetes de prensa «fomenta el periodismo perezoso y la información homogénea».

Esta práctica periodística de utilizar los comunicados de prensa de Nature y Science como fuentes informativas deriva en otros muchos problemas como la lejanía de las fuentes o la publicación de investigaciones que poco o nada aportan a la sociedad española. Sin embargo, una de las disfunciones más relevantes, puesta de manifiesto por el estudio ya citado del Observatorio de Comunicación Científica de la Universidad Pompeu Fabra de Barcelona, es que existe una relación directa entre la selección de los artículos para formar parte de los comunicados de prensa y sus posibilidades de ser difundidos en prensa. Es decir, la selección que hacen los periodistas de los gabinetes de comunicación de Nature o Science no está en función de los criterios de calidad científica, sino de los de noticiabilidad, sobre todo en el caso de Nature, que pertenece a una empresa privada. Esta relación, así como la sumisión de los medios a los comunicados de prensa de Nature o Science, es tal, según el estudio citado, que no sólo tienen posibilidades de ser mencionados por la prensa aquellos artículos seleccionados para los comunicados de prensa, sino que el orden en el que aparecen en dichos comunicados las investigaciones también influye decisivamente en su difusión en la prensa. Así, los artículos que figuran en primera o segunda posición en el comunicado de prensa son los que alcanzan una mayor difusión en los periódicos. Este estudio de los investigadores catalanes demuestra que los responsables de la agenda periodística son, en primer lugar, los que elaboran los comunicados de prensa de las revistas y, después, los periodistas de los diarios que los utilizan.

7 Sería interesante llevar a cabo una investigación sobre las diferencias entre los distintos comunicados de prensa, pero una simple observación de los de Nature pondrá de manifiesto hasta qué punto se identifican con los periodistas. En estos comunicados aparece primero una buena entradilla, típica de la estructura informativa de pirámide invertida, de forma que la noticia pueda ser copiada literalmente en las agencias o leída en informativos de radio y televisión. Luego introducen la investigación con una anécdota del científico principal o del proceso de investigación o con una metáfora, de forma que pueda comenzarse por ahí si se trata de escribir un reportaje. Todo son facilidades para el periodista.

${ }^{8}$ Entrevista de Gemma Revuelta a Lawrence Altman, revista Quark, Ciencia, Medicina, Comunicación y Cultura, n. ${ }^{\circ}$ 9, octubre-diciembre 1997 (pp. 75-77). 
Debo aclarar que en este trabajo no se ha analizado este fenómeno en diarios españoles porque, entre otros motivos, no dispuse de los comunicados de prensa del año 1998. Pero sí estoy en condiciones de afirmar que las noticias procedentes de Science y, sobre todo, de Nature se repetían en los mismos días en todos los medios, lo que induce a pensar que el fenómeno de Agenda Setting, según el cual son los medios de comunicación los que imponen qué es noticia y qué no, generado en el caso del periodismo científico por los editores de las revistas científicas de referencia, también se produce en España.

\section{INFLUENCIA DE LAS REVISTAS ESPECIALIZADAS EN LA «NOTICIA ACATAMIENTO»}

Otra disfunción importante respecto a la cada día mayor dependencia de los medios de comunicación de las revistas científicas es lo que el ensayista italiano Furio Colombo ha denominado "noticia acatamiento»". Este fenómeno, señala Colombo, se produce cuando la noticia llega a las redacciones con un plus de seguridad tal que el periodista baja la guardia de la comprobación y es fácilmente objeto de instrumentación de intereses ocultos. Colombo indica que este fenómeno, «que supone un riesgo muy importante para el futuro del periodismo», se da en todas las áreas del periodismo, pero considera que el paradigma del mismo es la noticia científica.

En el caso de las revistas científicas, este fenómeno se incrementa respecto de las noticias filtradas por los gabinetes de prensa oficiales — que también gozan de un plus de seguridad tal que los periodistas no las contrastan- puesto que la mayoría de los trabajos científicos publicados por Nature o Science tiene como autores a investigadores extranjeros.

Lo normal es que el plus de seguridad y credibilidad que poseen estas revistas provoca que el periodista acate sin más lo que en ellas se afirma. En el caso de querer contrastar la información, si no es un medio muy poderoso, lo tiene muy difícil. Si quiere acceder a la fuente informada - la que ha escrito el artículo_- lo normal es que esté saturada de periodistas y prefiera hablar con redactores estadounidenses o de su país de origen que con periodistas españoles, los cuales puede que no se expresen correctamente en inglés. Pero si opta por validar esos resultados con una fuente española de solvencia en ese campo, se encuentra con que el periodista - que recibe el informe embargado con una semana de antelación — tenga más información que el propio científico, igno-

9 Este fenómeno lo ha descrito Furio Colombo en su libro Últimas noticias sobre periodismo (Ed. Anagrama, Barcelona, 1997), en el que esboza cuál es, en su opinión, el futuro que le espera al periodismo en el mundo. El libro presenta un interesante capítulo dedicado al periodismo científico (pp. 96-111) en el cual señala que «la noticia científica viaja en periodismo con un inmenso valor añadido, aunque con riesgos importantes». 
rante del trabajo que Nature publicará una semana más tarde y que, normalmente, su contenido específico supone un secreto.

Todos estos problemas suceden en el mejor de los casos, el que supone que se dispone de ganas y tiempo para acometer el trabajo de contrastar la información. Cuando se trata de una noticia en caliente, que tenemos que escribir en unas pocas horas, esta tarea resulta imposible.

No critico tanto el hecho de que no se contrasten las informaciones - que es grave pero que a veces puede estar permitido- sino, sobre todo, que no se haga referencia a que no se han contrastado y se publiquen las dificultades.

\section{REPERCUSIONES FUTURAS DE LA WEB «EUREKALERT»}

Existe un tesoro muy bien guardado por los periodistas científicos veteranos de los diarios nacionales españoles y del resto del mundo. Este tesoro es la dirección en Internet http://www.eurekalert.org/e-infolabout.html. Esta web es un sistema semipúblico de noticias elaborado por la Asociación Americana para el Avance de las Ciencias y que utiliza el soporte técnico de la Universidad de Stanford. A ella estaban suscritos, en el momento de redactar este artículo —finales de 2000—, 2.058 periodistas científicos pertenecientes a 850 medios de comunicación de todo el mundo. También pertenecen 836 organizaciones de investigación de todo el planeta; a través de esta web puede accederse a cientos de revistas científicas internacionales. El departamento de prensa de esta web distribuye diariamente, entre los 2.058 periodistas abonados, una media de 22 comunicados. A través de ella se puede acceder a los artículos y comunicados de prensa de las revistas especializadas más importantes del mundo. Desde Cell, Nature ${ }^{10}$, Science, Neuron hasta las médicas como The Lancet o New England Journal of Medicine.

Prácticamente con esta dirección en Internet y conociendo el inglés, puede afirmarse que a un periodista científico no se le escapa ningún asunto relevante de la ciencia mundial.

Cuando uno accede, a través de Internet, a la lista de los 850 medios de comunicación suscritos a ella, llama la atención que, por parte española, sólo están los grandes medios: Radio Nacional de España, El País, El Mundo, Abc, El Periódico de Cataluña, La Vanguardia, Canal Plus y las agencias Efe y Europa Press. Pero no están los medios pequeños, los diarios regionales, muchos de los cuales tienen suplementos de ciencia.

${ }^{10}$ En teoría, se puede acceder a Nature porque en la dirección indicada aparece un enlace hacia esta revista. Sin embargo, en la práctica diaria, la revista Nature no incluye todos sus comunicados de prensa en el sitio Eurekalert. Muchas semanas no aparece ninguno. Una posible explicación a este comportamiento sería que Nature prefiere enviar sus comunicados de prensa directamente a los periodistas, pues se evita así la competencia con otras revistas. Debe tenerse en cuenta, además, que la dirección Eurekalert pertenece a la Asociación Americana para el Avance de la Ciencia, cuya publicación, Science, es la gran competidora de Nature. 
Los criterios de admisión sólo señalan que debe acreditarse la condición de periodista y que el medio al que pertenece, mediante declaración jurada, se comprometa a mantener las leyes de la información embargada. No dice nada acerca de si el medio debe tener o no una determinada difusión. Para evaluar la admisión o si el comportamiento ético en cuanto a la aceptación de las condiciones de embargo es el correcto, existe un comité compuesto por periodistas científicos y por representantes de las revistas que en este sitio web aparecen.

En un principio, quien suscribe este artículo pensó que el hecho de que no existieran periódicos regionales tipo El Correo Vasco, El Heraldo de Aragón o La Voz de Galicia, entre otros, se debía a que entre los criterios de admisión se establecía la difusión del medio. Pero esto no tiene sentido porque la distribución de información a través de Internet es instantánea y no repercute el número de abonados. Tras conversar con algunos de los que no están en la lista, señalaron que no habían solicitado ser incluidos porque no necesitan una información tan especializada.

También destaca la escasa presencia de diarios latinoamericanos. Hay algunos importantes: Clarín o El Mercurio, entre otros, pero faltan muchos.

El acceso de los medios de comunicación a los embargos de Eurekalert es totalmente gratuito. No obstante, deben respetarse escrupulosamente las leyes del embargo, pues en caso contrario el medio de comunicación puede, a criterio del comité, resultar eliminado de la lista durante un periodo que puede ser, incluso, indefinido.

Otro dato interesante respecto a esta dirección es que muchas veces los comunicados de prensa que aparecen en ella no pertenecen a los artículos más relevantes de la revista en cuestión. La impresión de los periodistas consultados es que, muchas veces, los comunicados son elaborados por las propias universidades a las que pertenece la investigación.

Además de estos comunicados de prensa, en Eurekalert aparecen resúmenes de cinco líneas de los artículos publicados en las revistas. Muchas agencias, entre ellas Europa Press, traducen estas noticias y las difunden a los abonados como embargadas a primera hora de la mañana del día en que finaliza el embargo.

La agencia Reutes también suele enviar los resúmenes, pero tras ellos incorpora grandes reportajes en los que ha hablado con los científicos que han hecho la investigación. Por tanto, esta agencia también puede considerarse como una de las principales fuentes de cualquier periodista científico.

Internet ofrece asimismo la posibilidad de acceder al sitio web de la cadena de televisión británica $B B C$, considerada por los especialistas como una de las que más y mejor información ofrece sobre los acontecimientos científicos.

En un futuro habrá que analizar cómo afectará Internet a la internacionalización de la información científica en los medios de comunicación. Las ventajas son innumerables. Se tiene acceso a los principales centro de investigación del mundo, a debates entre periodistas científicos de todo el planeta o los 
acontecimientos científicos que se prevé celebrar con una antelación de un año. En realidad, cuando uno visita estas páginas o las de centros como la NASA, el CNRS francés o la Royal Society británica y las compara con las equivalentes del CSIC o de las universidades españolas, comprende, sin necesidad de explicaciones, a qué nivel nos encontramos.

Entre los efectos negativos, preveo una excesiva internacionalización de la información, la dependencia de Estados Unidos, así como de los comunicados de prensa que se reciben diariamente a través de esta web.

\section{EFECTOS PERVERSOS DE LA PRENSA EN LA CIENCIA DEL FUTURO}

Los científicos son cada día más conscientes de la importancia de que sus investigaciones sean publicadas en los medios de comunicación. Una de las formas más seguras para conseguir este objetivo es que sus trabajos aparezcan en revistas como Nature o Science. Pero, como se tratará de demostrar más adelante, los parámetros que exigen estas publicaciones para admitir artículos no sólo dependen de su calidad científica. Esta circunstancia podría provocar que se modifique la dinámica investigadora de forma que se primen en ella los condicionantes exigidos por estas revistas en vez de los requeridos por la sociedad o la propia ciencia. Respecto a la ciencia española, estos efectos perversos son fundamentalmente dos: la desaparición del español como idioma para comunicar la ciencia y el fenómeno de producir ciencia diseñada exclusivamente para que sea publicada en los periódicos, desdeñando otras prioridades. Este último caso puede generalizarse al resto del mundo.

\section{LA DESAPARICIÓN DEL ESPAÑOL COMO IDIOMA PARA COMUNICAR LA CIENCIA}

Antes de explicar este fenómeno, cuyas causas directas tienen que ver con la creciente importancia en términos de impacto de Nature y Science, no sólo en cuanto a citas en otras publicaciones, sino también en su presencia en la prensa, debo aclarar que en ciencias experimentales el índice de impacto es fundamental a la hora de conseguir una plaza de investigación, no sólo en España sino en el resto del mundo. Un artículo publicado en Nature cuyo SCI es de $28,833^{11}$ es multiplicado por ese coeficiente en el caso de que su autor se presente a cualquier concurso de méritos. Es decir, que vale más publicar un artículo en Nature que 28 en revistas con impacto de uno -que son la gran mayoría-; y no digamos nada de aquellas cuyo impacto no llega a la unidad. Publicar en una con impacto 0,5 es como tener la mitad de un artículo. Para

11 Datos referidos al último SCI publicado y que corresponde a 1998. 
comprender la importancia de Nature o Science baste decir que la revista que ocupa el cuarto lugar en la clasificación del índice de impacto de revistas multidisciplinarias, Bioscience, tiene un índice de 2,983. Un artículo en Nature vale más que nueve en Bioscience. Esta clasificación SCI, que se publica de forma periódica desde los años setenta, provoca que los grandes trabajos se presenten en Nature o Science y que cada día estas dos publicaciones se separen más del resto de las revistas en cuanto al índice de impacto y, aunque generalistas, se vayan acercando cada día más al índice de impacto de los abstracts, con lo cual su dominio del mundo científico será casi absoluto dentro de unos años.

Este índice de impacto se calcula en una institución privada de Filadelfia (EE.UU.), teniendo en cuenta, entre otros factores, la cantidad de veces que un artículo aparecido en la revista es citado por otras publicaciones. A esa cifra se le aplica asimismo un coeficiente derivado del índice de impacto de las revistas en donde ha sido citado el artículo. Tras la elaboración de este coeficiente, seguido con distintos grados de aceptación en todas las universidades y centros de investigación del mundo, todas las revistas científicas de países como España, Francia, Alemania o Japón deben ser publicadas en inglés si quieren tener alguna posibilidad de que sus artículos sean citados en alguna de difusión internacional. Este índice ha provocado asimismo determinadas disfunciones como que los investigadores de esos países, cuando tienen un buen trabajo, lo envían a las publicaciones de gran impacto, por lo que las nacionales no reciben buenas investigaciones y cada vez son menos citadas. La rueda lleva a que se desprecien esas otras revistas y que, por ejemplo, revistas científicas en español, de tradición centenaria, tengan índice de impacto cero - porque no aparecen en el SCI_, con lo cual publicar en sus páginas no valga como mérito ni para una plaza en España. Esto ha provocado que la presencia del idioma español en la difusión científica mundial sea totalmente nula ${ }^{12}$.

El presidente desde 1985 de la Real Academia Española de Ciencias Exactas, Físicas y Naturales, Ángel Martín Municio, aseguró a quien suscribe este artículo $^{13}$ que esta política de minusvalorar el idioma español en la producción científica comenzó en 1985 y los responsables, a su juicio, son los gobernantes socialistas de la época, y en especial Juan Rojo, quien decidió, dijo Martín Municio, que la producción científica española se evaluara, a la hora de valorar los méritos para percibir retribuciones en función de esa producción, teniendo como base el impacto SCI.

${ }_{12}$ Un artículo del presidente de la Real Academia Española de Ciencias, Ángel Martín Municio, publicado en el Anuario correspondiente a 1998 del Instituto Cervantes, incide en que desde hace unos años la presencia del idioma español en la ciencia mundial, en vez de aumentar, tal y como lo hace su número de hablantes, ha descendido a tales niveles que puede afirmarse que su presencia en publicaciones o congresos científicos relevantes es prácticamente nula.

13 Quien suscribe este artículo ha entrevistado en varias ocasiones al presidente de la Academia de Ciencias, unas como periodista y otras como investigador. 
«A partir de ese año —explicó Martín Municio- ningún científico español quiso publicar en revistas españolas porque éstas no estaban incluidas en la selección que hace una empresa privada radicada en Filadelfia pero que nuestro gobierno de turno eligió como referencia. Revistas centenarias como los Anales de Quimica donde publicaron sus trabajos científicos de la talla de Echegaray o Blas Cabrera, dejaron de tener importancia porque se publicaban en español. Lo paradójico es que con tanta defensa del español que quieren hacer ahora, resulta que en 1999 se publica menos ciencia en español que a principios de siglo. Todo por la falta de visión política que no es sólo socialista, porque el PP ha copiado literalmente el decreto que premia la producción científica en función del índice SCI. Como alguien no tome medidas drásticas, desaparecerán todas las que aún subsisten. Pero a nadie le importará, porque la ciencia nunca les ha interesado a los políticos españoles».

Posibles políticas que potencien el idioma español en la ciencia serían, por ejemplo, valorar las publicaciones en esa lengua, establecer revistas en el ámbito latinoamericano y potenciar que éstas dispongan de gabinetes de prensa potentes como las anglosajonas, de manera que las investigaciones que en ellas se publiquen lleguen en forma de comunicado a todos los medios de comunicación del mundo. No son decisiones costosas sino políticas, pero quizá ésa sea la principal dificultad en un país como España.

En cualquier caso, no incidiré más en este asunto que merece un estudio más detallado por las autoridades y de los estudiosos, pero que no es parte fundamental de este trabajo.

\section{LA CIENCIA MEDIÁTICA: EL FENÓMENO DE LA CIENCIA PRODUCIDA EXCLUSIVAMENTE PARA SALIR EN LOS PERIÓDICOS}

A estas disfunciones se les agrega el hecho de que, desde hace unos años, los artículos científicos que son publicados por las revistas Nature o Science pero, sobre todo, que son difundidos por los medios de comunicación, son mucho más citados por los propios científicos que los que simplemente se publican en una revista de prestigio.

Esta circunstancia ya fue demostrada en 1991 en un estudio publicado en New England Journal of Medicine ${ }^{14} \mathrm{y}$ en el cual sus autores (Phillips et al.) analizaron los artículos de la citada revista publicados en 1978 y 1979, y compararon los que aparecieron en The New York Times con los que no lo hicieron. Observaron que los estudios que se habían publicado en el periódico estado-

${ }^{14}$ Estudio citado por Robert Finn en su artículo «Por qué vale la pena la comunicación», reproducido en la revista Quark, Ciencia, Medicina, Comunicación y Cultura, n. ${ }^{\circ}$ 10, enero-marzo 1998 (pp. 47-57). 
unidense habían sido citados un $72,8 \%$ más en el año siguiente a su publicación que los que no aparecieron en ese diario. Lo más relevante es que la diferencia significativa de citas persistió durante al menos diez años tras la publicación de los resultados científicos en el periódico.

El citado estudio incluía un control muy bien elaborado para comprobar la suposición razonable de que The New York Times sólo estaba ofreciendo información sobre los estudios más significativos, que obviamente habrían recibido más citas. Sin embargo, durante el periodo estudiado The New York Times sufrió una huelga de tres meses (1978). Durante la misma el periódico produjo sus números, que quedaron en una "edición registro", aunque ninguno de ellos se distribuyó al público. Los autores del trabajo analizaron esta "edición registro", por lo que quedaba claro qué artículos consideraba The New York Times dignos de cobertura aun cuando no se estuvieran publicando. El estudio demostró que esos artículos dignos de publicación, pero no publicados por causa de la huelga, no fueron objeto de un aumento de la cantidad de citas refiriéndose a ellos en los años siguientes. De forma que quedó demostrado que un artículo científico que es mencionado en la prensa de calidad es citado un $78,2 \%$ más, independientemente de la calidad de la investigación.

Como además el índice de impacto de la revista se mide, como hemos mencionado, en función de cuántas veces han sido citados sus artículos en otras publicaciones, si la revista consigue colocarlos en la prensa, sabrá que para el año siguiente obtendrá un mayor índice SCI, lo que implicará que los mejores científicos querrán publicar en ella, por lo que esta dinámica circular se incrementará a su favor.

Pero la distorsión se produce porque sólo Nature y Science y algunas revistas médicas poseen agresivos gabinetes de prensa, de forma que cada día estas publicaciones tendrán mayor índice de impacto y el resto se quedará irremediablemente en puestos muy inferiores.

Esto, con ser un problema serio, no sería tan grave si no fuera porque la prensa internacional, incluida la española, está ayudando a que las revistas como Science y, sobre todo Nature sean las que tienen un mayor índice de impacto.

Debe aclararse que estas publicaciones son generalistas y que su objetivo principal no persigue favorecer la ciencia per se, sino ganar y agradar a sus lectores y anunciantes, pues viven de eso. Esto provoca que muchas veces sean criterios de noticiabilidad los que imperan a la hora de seleccionar sus artículos. En este sentido, el artículo publicado por Nature el 4 de enero de 1996 sobre los efectos analgésicos de la mirra es un caso paradigmático. El artículo era de relevancia científica mínima, pero se publicó en la semana de Reyes. El estudio de Nature tenía un título típicamente descriptivo de los artículos científicos pero, en el comunicado de prensa, Nature titulaba el trabajo: "Por qué los tres Reyes Magos llevaban mirra». Obviamente, la noticia fue seleccionada por muchos medios de comunicación porque se adaptaba perfectamente a la actualidad de la semana. Primero fueron los periódicos y, después, como suele 
suceder en el periodismo científico, se hacen eco de la noticia las emisoras de radio y televisión.

Esta gran cobertura de temas irrelevantes y el aumento de citas que llevan consigo tras ser publicados en la prensa hará que, en el futuro, muchos científicos estudien efectos colaterales de la ciencia que son mucho más noticiables que la ciencia básica. Cientos de científicos que en el mundo investigan los efectos analgésicos de diversas sustancias mencionarán en sus referencias la relativa a la mirra publicada por Nature, de forma que un artículo en principio irrelevante se convertirá en importante y sus autores ganarán méritos con el actual sistema de evaluación de investigadores.

Esto provocará que otros científicos, debido a la creciente competitividad a la que están sometidos, desistirán de investigar temas tediosos y con pocas perspectivas de convertirse en noticias, aunque sean relevantes desde el punto de vista del corpus científico, y dirigirán sus estudios a efectos colaterales de los mismos. El estudio de lo que implicaría el impacto de un meteorito en la Tierra, la sustancia química responsable del enamoramiento, la estrella que guió a los Reyes Magos o la posible presencia de componentes anticancerígenos en los más peregrinos alimentos, desde el vino y las uvas hasta los aceites de oliva o los chocolates, se convertirán en temas adecuados para investigarlos, desechando otros más tediosos.

Con el actual sistema de evaluación de científicos, los que investigan los temas colaterales resultan mejor valorados que sus colegas que han optado por abordar asuntos relevantes pero menos noticiables. Estos últimos, defraudados por las escasas expectativas de que sus resultados se publiquen en los medios de comunicación, abandonan sus investigaciones por otras más efectivas desde el punto de vista periodístico. En qué medida la ciencia mundial sale perjudicada por estos fenómenos surgidos en estos últimos años es algo que escapa al objeto de este estudio, pero creo que debería analizarse de una forma más seria. Tal vez la solución estaría en valorar menos el índice de impacto y buscar otros sistemas para evaluar el rendimiento de los científicos. Pero esta circunstancia, reitero, no es objeto de este trabajo.

\section{BIBLIOGRAFÍA}

AzUARA Solís, José Ángel (1990): «Información científica: un cambio de actitud», en Actas del I Congreso de Periodismo Cientifico (pp. 43-48), CSIC, Madrid.

Bunge, Mario (1985): Seudociencia e ideología, Alianza Editorial, Madrid.

Burhnam, John C. (1988): How Superstition Won and Science Lost: Popularizing Science and Health in the Unit States, Rutgers University Press, Nueva Yersey.

Burkett, David W. (1965): Writing Science News for the Mass Media, Gulf Publishing Company, Houston.

- (1986): News Reporting: Science, Medicine and High Technology, The Iowa State University Press, Iowa. 
Chaparro, Manuel Carlos (1990): «De la ciencia al pueblo por la vía periodística», Arbor, Ciencia, Pensamiento y Cultura, n. ${ }^{\text {s }}$ 534-535, junio-julio (pp. 43-59), CSIC, Madrid.

Colombo, Furio (1997): Últimas noticias sobre periodismo, Anagrama, Barcelona.

CoRnell, James (ed.) (1986): The International Popularization of Science, International Science Writers Association, Cambridge, MA.

Deason, Hilary, J. A. (1963): Guide to Science Reading, The American Association for the Advancement of Science, Nueva York.

ELÍAs, Carlos (2001a): «Estudio cuantitativo de las fuentes en el periodismo español especializado en ciencia", Revista Latina de Comunicación Social, n. 38 (febrero), D-L: TF-135-98/ ISSN: $1138-5820$.

- (2001b): «Periodismo especializado en medio ambiente: el caso Doñana como paradigma de manipulación informativa», Ámbitos: Revista Andaluza de Comunicación, n.o 7 (junio) (pp. 297-303).

Evans, William, y Hornin Priest, Susanna (1995): «Science Content and Social Context», Public Understanding of Science, n. ${ }^{\circ} 4$ (pp. 327-340), Londres.

Franklin, Jon (1998): "El fin del periodismo científico», Quark, Ciencia, Medicina, Comunicación y Cultura, n. ${ }^{\circ}$ 11, abril-junio (pp. 53-63), Universidad Pompeu Fabra, Barcelona.

Friedman, S. (1986): Sciences and Journalists: Reporting Science as News, Free Press, Nueva York.

Friedman, Sharon; Dunwoody, Sharon, y Rogers, Carol (1986): Scientist and Journalists: Reporting Science as News, The Free Press, Nueva York.

Jensen, K. B., y JANKOWSKY, N. W. (1993): Metodologías cualitativas de investigación en comunicación de masas, Bosch-Comunicación, Barcelona.

Montaño, Miguel (1999): «El Canal 2 de Andalucía y la información ambiental: el programa Espacio Protegido", Ambitos: Revista Andaluza de Comunicación, n. ${ }^{\circ}$ 2, enero-junio (pp. 207228), Universidad de Sevilla.

Nelkin, Dorothy (1987): Selling Sciences. How the Press Covers Science and Technology, Freeman, Nueva York (edición española: La ciencia en el escaparate, Fundesco, Madrid, 1990).

Pérez Mercader, Juan (1990): «Las ventajas de la relación entre científicos y periodistas», en Actas del I Congreso de Periodismo Científico (pp. 151-154), CSIC, Madrid.

PÉrez Oliva, Milagros (1998): "Valor añadido de la comunicación científica», Quark, Ciencia, Medicina, Comunicación y Cultura, n. ${ }^{\circ}$ 10, enero-marzo (pp. 58-69), Universidad Pompeu Fabra, Barcelona.

SAPERAS, Enric (1987): Los efectos cognitivos de la comunicación de masas: las recientes investigaciones en torno a los efectos de la comunicación de masas: 1970-1986, Ariel-Comunicación, Barcelona.

Schatzman, L., y Strauss, A. L. (1971): Field Research: Strategies for a National Sociology, Englewood Clift, Nueva Jersey.

SCHMERTZ, Herb, y NovAK, William (1986): El silencio no es rentable. El empresario frente a los medios de comunicación, Planeta, Barcelona.

SNOw, C. P. (1965): El conflicto de las dos culturas, Proezas del Pensamiento, México.

VelázQueZ, Roberto (1990): «Las dificultades de la comunicación científica», Arbor, Ciencia, Pensamiento y Cultura, n. ${ }^{\text {s }}$ 534-535, junio-julio (pp. 115-122), CSIC, Madrid.

WOLF, Mauro (1996): La investigación en la comunicación de masas: críticas y perspectivas, Paidós, Barcelona, tercera reimpresión de la obra editada en 1987. 


\begin{abstract}
In this study, an attempt has been made to detect the influence of impact magazines in which the majority of the articles published are written by scientists, on specialist journalism and on present-day science. I have analysed the Spanish case in particular, but I consider that its conclusions can be extended to all other countries. Apart from the fact that the absolute preponderance of magazines such as Nature and Science is demonstrated: 45 per cent of the information on science given by foreign scientists who publish their work in generalist newspapers is taken from these magazines, also analysed are the influences that the media communication strategy used by the press offices of these publications exert on science. A special study has been made of the preponderance of English and the scanty relevance of Spanish, besides the future consequences that the ever-increasing impact of a few magazines will have on modification of the scientific model in experimental sciences.
\end{abstract}

